\title{
STAND-ALONE PROGRAM PACKAGE FOR IMPROVEMENT OF PARAMETERS OF CELESTIAL BODIES MOTION
}

\author{
T. V. IVANOVA, F. A. NOVIKOV and E. Yu. PARIISKAYA \\ Institute of Theoretical Astronomy of the Russian Academy of Sciences, St.Petersburg \\ E-mail ita@iipah.spb.su
}

A stand-alone Program Package for Improvement (PPI) of motion parameters of celestial bodies from observations is described. The PPI is oriented for solving conditional equations system by the method of least squares and is implemented for IBM PC in MSDOS in the Turbo-Pascal programming language v.5.0. There are two versions of the system:

A) using the standard conventional memory $640 \mathrm{~Kb}$ and

B) using the external memory.

About 250 parameters can be improved by means of the version A simultaneously. In the version $\mathrm{B}$ the maximum number of the improving parameters is much greater and is limited only by the amount of the external memory available.

The most important feature of the PPI is a special improvement language with wide range of facilities for the presentation of input and output data and modes of improvement process. This language allows to set an iterative process of improvement on the $n \sigma$ criteria (where $n$ is a real number and $\sigma$ is a standard deviation) and to repeat the improvement process with different subsets of unknowns as well. In the case of non-intersecting subsets of unknowns it gives a possibility of independent improvement of parameters with different physical meaning in one task (for example, global and local parameters for each series of observations).

The PPI package can treat simultaneously observations of any kind (optical, radiointerferometric, laser etc.) using scale factors for different observed data. The PPI allows to improve parameters for unlimited number of observations due to its ability of accumulation of the normal system in several consequent tasks. The observations themselves should be provided as files together with information on conditional equations coefficients. It is possible to modify and set new coefficients by means of usual mathematical formulas directly in the task not changing the source data. The PPI package allows to reject observations depending on various conditions. Furthermore each group of observations might be supplied with its own weight function.

The package discussed is the further development of our package described in Ivanova and Novikov (1990).

\section{References}

[1] Ivanova T.V. and Novikov F.A. (1990) Program Package for Improvement of Parameters of Equations of Condition for IBM PC, Preprint of the Institute of Applied Astronomy of the USSR Academy of Sciences (Leningrad), 21 (in Russian). 